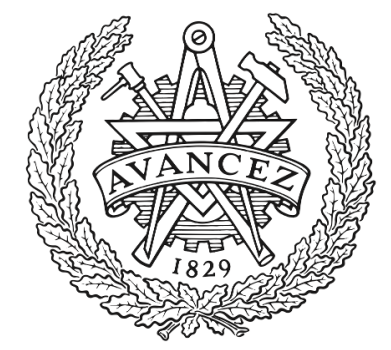

\title{
CHALMERS
}

UNIVERSITY OF TECHNOLOGY

\section{Chalmers Campus as a Testbed for Intelligent Grids and Local Energy Systems}

Downloaded from: https://research.chalmers.se, 2023-04-26 09:15 UTC

Citation for the original published paper (version of record):

Antoniadou-Plytaria, K., Srivastava, A., Fotouhi Ghazvini, M. et al (2019). Chalmers Campus as a Testbed for Intelligent Grids and Local Energy Systems. IEEE International Conference on Smart Energy Systems and Technologies (SEST). http://dx.doi.org/10.1109/SEST.2019.8849014

N.B. When citing this work, cite the original published paper. 


\title{
Chalmers Campus as a Testbed for Intelligent Grids and Local Energy Systems
}

\author{
Kyriaki Antoniadou-Plytaria, Ankur Srivastava, Mohammad Ali Fotouhi Ghazvini, David Steen, \\ Le Anh Tuan and Ola Carlson \\ Division of Electric Power Engineering \\ Department of Electrical Engineering \\ Chalmers University of Technology \\ 41296 Gothenburg, Sweden \\ Email: \{kyriaki.antoniadou, ankur.srivastava, ali.fotouhi, david.steen, tuan.le, ola.carlson $\} @$ chalmers.se
}

\begin{abstract}
This paper presents an overview of a testbed for intelligent distribution grids, local energy systems, and energy flexible buildings, which is being developed at the campus of Chalmers University of Technology in Gothenburg, Sweden. It describes the test sites, the functionalities, and the planned demonstration activities within the scope of on-going research projects. The proposed demonstrations include a local energy market platform, energy management solutions for microgrids and smart buildings, as well as voltage control in distribution grids. The paper aims to show how the physical energy supply systems of the university are being adapted to integrate the communication and control set-ups that provide the technical requirements for smart grid interoperability. As an example, the on-site implementation of remote battery control is presented, where initial results show the feasibility and potential benefits of the external control. Finally, challenges and lessons learned during the development of the testbed are highlighted.
\end{abstract}

Index Terms-Campus microgrid, distribution network, energy management system, intelligent grids, microgrids, smart buildings, smart grid.

\section{INTRODUCTION}

Research on intelligent grid solutions has proliferated over the past years to address the uncertainties introduced at the distribution level due to intermittent generation and plug-in electric vehicle (PEV) charging. The real-site employment of these solutions, however, often has to deal with various technical issues such as interoperability between products of different vendors or errors due to average modeling of certain system parts. Engagement of the associated stakeholders and coordination among them can also be challenging.

The need to bridge the gap between research and deployment motivated the development of demonstration sites such as microgrids [1]-[5] and living labs [6]-[8]. These sites allow the researchers to gain hands-on experience on the communication interfaces of data acquisition and transmission and apply their knowledge to improve the efficiency and

This work is financially supported by: i) The joint programming initiative ERANet Smart Grids Plus under grant agreement No 646039, ii) Göteborg Energi AB (GEAB) under the project SN11 "Innovative energy management system for smart buildings and grid interactions", iii) Urban Innovative Actions program to the Fossil Free Energy Districts (FED) project (No. UIA01209), iv) The European Community's Horizon 2020 framework programme under grant agreement no. 773717 robustness of their models. Demo sites at university campus areas in particular [1], [6] provide unique opportunities to attract collaboration with industry and leverage research. In addition, the size of a university campus can more easily accommodate a physical demonstration unlike large-scale systems.

For these reasons, demonstration sites are also developed in the Chalmers University of Technology campus. These sites are expected to validate and increase the technology readiness level (TRL) of the smart grid solutions associated with different research projects. The campus facilities provide many benefits to the on-going research because they incorporate up-to-date measurement and control equipment. Moreover, the campus represents a real-life environment suitable for credible case studies. The support of the local distribution system operator (DSO), named Akademiska Hus, is also an essential contribution as the engagement of the stakeholders is extremely important for the conduction of real-site tests.

This paper focuses on the development of physical demonstration sites for smart grid and smart building implementations at the Chalmers campus in Gothenburg. It presents the technical details of the test sites and describes the smart grid functionalities, which are being tested under the following projects: $m 2 M-G R I D$ [9], FED [10], UNITED-GRID [11], and $B E M S$ [12]. The demonstration activities, which are linked to these projects include energy management systems (EMS) for microgrids and buildings, microgrid interfaces, coordinated voltage control, and a local multi-energy market.

The main contribution of this paper is to indicate how a physical energy system can be employed to study energy system functionalities. For this purpose, the related information and communication technology (ICT) infrastructure and the recently developed interface set-ups are presented to provide valuable insight to the requirements, challenges and potential benefits of the future demonstration tests.

The rest of the paper is structured as follows: The technical characteristics of the test sites are described in Section II. Sections III-IV discuss the smart grid functionalities and the demonstration cases of the projects, respectively. Section $\mathrm{V}$ presents an example of a demonstration test. Section VI comments on the challenges of preparing the testbed and finally, conclusions are discussed in Section VII. 


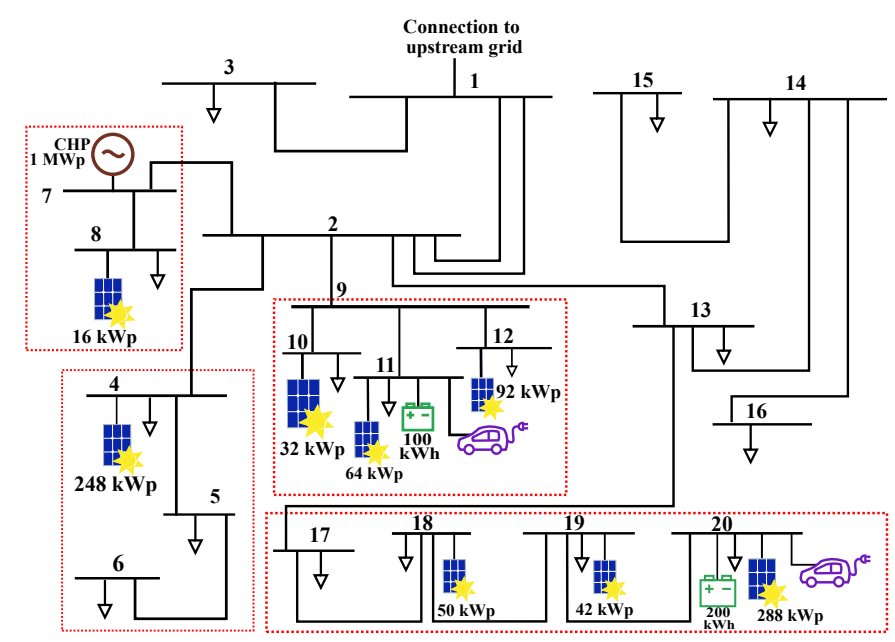

Fig. 1. The distribution network of Chalmers. The dotted lines include network areas that can be considered as grid-connected microgrids.

\section{TEST SiteS}

The first test site described in this section is the electrical distribution network under the secondary substation that supplies Chalmers campus's buildings. The other test site is a residential building inside the campus area (connected to a different secondary substation), which was constructed primarily to support research and also to accommodate students.

\section{A. Electrical Distribution System of Chalmers Campus}

The $12-k \mathrm{~V}$ distribution network of Chalmers (Fig. 1) has a total electrical load demand that varies between 2.5 and $6 \mathrm{MW}$ [13]. The load demand is supplied by importing energy from the upstream distribution grid and, in addition, by electricity generated through a micro-combined heat and power (CHP) plant and solar panels. The CHP plant consists of a boiler with 3-6 MW heating capacity, while the electric capacity of the steam turbine is $0.5-1.0 \mathrm{MW}$ (the heating or electrical operational power output depends on the choice of fuel).

The total photovoltaic (PV) capacity is $831 \mathrm{kWp}$ and the majority of the solar panels have been installed on rooftops of the campus's buildings, with only a few solar panels of $16 \mathrm{kWp}$ total capacity mounted on the wall of a building. The network also hosts two battery energy storage systems (BESS) with energy capacity of $200 \mathrm{kWh}$ and $100 \mathrm{kWh}$ and $35 \mathrm{PEV}$ charging points at $32 \mathrm{~A} / 22 \mathrm{~kW}$ and $16 \mathrm{~A} / 3.7 \mathrm{~kW}$ level, located at two different charging stations. The facilities include an advanced metering infrastructure (AMI) system that consists of an ABB MicroSCADA and smart meters. The buildings of Chalmers campus are also equipped with various energy meters as well as controllable devices that enable the building operator to control the heating, cooling, and ventilation system.

\section{B. HSB Living Lab}

Inside the campus area there is a multi-family residential building of 29 apartments, which is called HSB Living Lab (HSB LL) [14]. It is a unique testbed for sustainable living solutions, where the living lab approach focuses on applying innovation in human-centered systems.

In 2018, the solar panels installed on the facade and the roof of HSB LL, produced 10.82 MWh of energy in total, while the annual electricity consumption of the building was $70.7 \mathrm{MWh}$ (the daily demand ranged between $61 \mathrm{kWh}$ and $259 \mathrm{kWh}$ ). The $18 \mathrm{kWp}$ PV system is coupled with a $7.2 \mathrm{kWh}$ battery, which can be charged both from the PVs and the AC grid. The PV and BESS system is connected to the AC grid via a converter provided by Ferroamp.

Apart from the installed distributed energy resources (DER), an advanced metering and sensor system is deployed at the building, which includes approximately 2000 sensors that collect various building data. This system is used to investigate the resident behavior's impact on energy consumption.

\section{FUNCTIONALITIES}

The demo sites can be employed to test the performance of various functionalities in the intelligent grid context. The functionalities that are briefly described in this section are:

- Congestion management

- Coordinated voltage control

- Microgrid interfaces

- Microgrid energy management system (MG-EMS)

- Building energy management system (BEMS)

- Load management

- Energy profiling

- Local energy market platform

In the smart grid era, multiple inverter-interfaced DER and controllable loads can be employed by the DSOs to participate in coordinated voltage control and congestion management so that the bus voltages and line currents are regulated in realtime within the acceptable limits. The flexible resources and various intelligent electronic devices (IEDs) can coordinate with the distribution management system (DMS) platform using online communication to assist the operation of the network by changing their operation set-points (e.g., active power injection, reactive power injection/absorption and voltage).

The implementation of microgrids can also relieve the DSO from the complex control tasks by efficiently coordinating producing and consuming units. The development of microgrid interfaces enables coordination not only among local resources but also among interconnected entities with the aim to facilitate resource sharing for improved network operation.

The microgrid interfaces can be classified in three categories: 1) microgrid interface, for control of the microgrid resources/components by the microgrid operator, 2) microgrid to DMS interface, for control of microgrid resources after coordination between the microgrid operator and the DSO, and 3) microgrid to microgrid interface, for control of the microgrid resources/components after interaction between the microgrids (e.g., energy trading, load sharing).

An integral part of the microgrid is the microgrid energy management system (MG-EMS), which is responsible for the real-time balance of supply and demand by dispatching active 
(or even reactive) power of generating resources, energy storage systems and flexible loads. There are multiple operational strategies that can be applied by a MG-EMS depending on the characteristics of the system and the objectives of the operator (e.g., cost savings, reduction of $\mathrm{CO}_{2}$ emissions, etc.).

The long-term goals of energy development in buildings are to maximize the efficiency of power consumption and reduce the losses, while the short-term goals are to reduce the peak demand and minimize the consumption during periods with high energy prices or fossil fuel-based power generation. A building energy management system (BEMS) is an ideal option to provide the short-term goals.

The BEMS focuses on advanced load management techniques, which use information exchange among users, appliances and controllers. Usually, the objective is to minimize the total energy cost of the building. Other objectives are to reduce the daily peak demand or the consumption fluctuations. A central issue in load management is the energy profiling, which is the characterization of operational patterns and energy consumption over time [15]. Discovery of the typical profiles, load classification as well as the customers' classification are different aspects of energy profiling, which can be accommodated by the AMI system of the smart buildings.

The purpose of a local energy market platform is to enable multiple actors (producers, consumers, prosumers) representing multiple energy carriers (electricity, heating, cooling) to trade their flexibility. Such a platform contributes to supply a local area with energy which is both low-cost and less dependent on fossil energy sources.

\section{DEMONSTRATIONS}

The deployed DERs, controllable devices and AMI system make the Chalmers campus a highly suitable demo site to test some of the previously mentioned intelligent grid functionalities, which are also linked to on-going research activities.

The interrelation of the project activities can be seen in Fig. 2. The preparation of the Chalmers testbed was initiated by the fossil-free energy districts (FED) project, which develops a local energy market for electric, heating, and cooling energy trade. This relates to the $B E M S$ project, where multi-energy carriers are utilized in an EMS for a smart building. The EMS integration is also a part of the $m 2 M-G R I D$ field tests. This project develops a test system that will contain an emulated MG-EMS for a grid-connected microgrid. The $m 2 M-G R I D$ and UNITED-GRID projects focus specifically on interactions with the electric distribution system enhancing the testbed of FED.

\section{A. FED: Local Energy Market Platform}

The FED project has been the driving force that initiated the demonstrations at Chalmers campus by developing automated digital solutions. Moreover, it financed the installation of a significant amount of solar generation capacity making big steps towards the creation of a fossil-free and sustainable university environment. In the FED project, market actors represented by agents participate in energy trade by bidding in a cloud-based local energy market. This digital market platform interacts

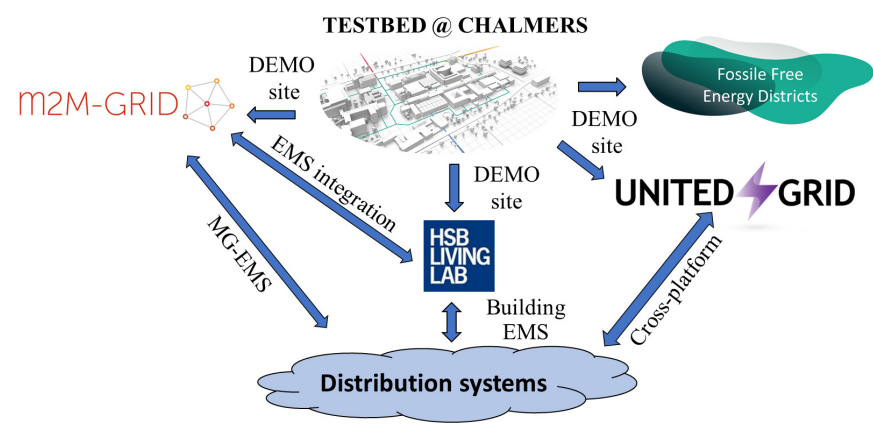

Fig. 2. Interaction between project activities.

with the on-site resources through a web-based HMI/SCADA interface. The local energy market does not only consider electricity but also heat and cooling, which makes it possible to capture any synergies between the different energy carriers.

\section{B. m2M-GRID: Grid-connected Microgrid EMS}

One of the main objectives of the $m 2 M-G R I D$ project is to propose interfaces for microgrid interoperability. The MGEMS will be a tool for the microgrid operator to schedule the microgrid's DER according to local operational strategies. Simultaneously, coordination can also be considered both with the upstream network and with other microgrids to supply/procure flexibility and engage in energy trade.

1) $M G$-EMS Demonstration: The $m 2 M-G R I D$ project aims at a microgrid demonstration at Chalmers distribution network, where certain network areas will be considered as gridconnected microgrids. Each microgrid will be represented by an emulated MG-EMS.

An optimal dispatch algorithm will use forecasted values of load and PV generation for the day-ahead and hour-ahead energy scheduling of the resources. The optimization objectives include cost minimization and minimization of energy exchange with the upstream network. Re-scheduling may also be decided if the MG-EMS receives flexibility requests by the DSO to increase or decrease the power at the point of common coupling (PCC), which is the physical interface of the microgrid with the distribution network.

If some data are not available, pseudo-measurements (e.g., short-term forecasted or historical values) can be introduced to solve the microgrid power balance or the upstream network power flow problem. Once the optimal decisions have been calculated, the MG-EMS will send the active power set-points to each of the microgrid resources.

2) Communication Set-up: The communication set-up establishes the connection of the microgrid resources to the server, where the MG-EMS will run. It facilitates the emulation of the MG-EMS, a procedure which requires that the data acquisition, the execution of optimization algorithms and the dispatch of set-points are performed in an automatic way.

The emulated MG-EMS will synchronize its operation with measurement and control systems using backend services (Fig. 3 ). The field and building devices and controllers are integrated to the web-based HMI/SCADA system via the Transmission 


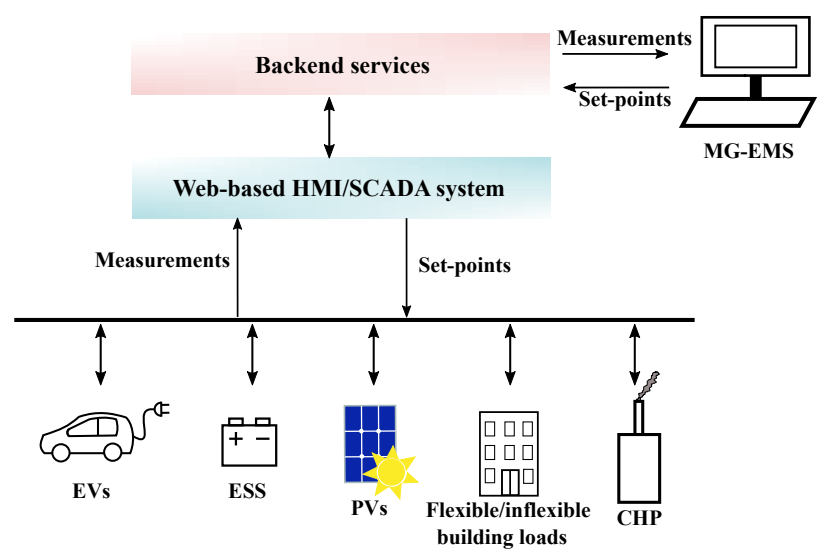

Fig. 3. Communication interface set-up for the microgrid energy management system in Chalmers.

Control Protocol/Internet Protocol (TCP/IP) or other protocols that run on top of it, such as the message queuing telemetry transport (MQTT) protocol and Modbus. Finally, the MG-EMS can interact with the resources through the SCADA with the help of web application programming interfaces (APIs) that $\mathrm{read} / \mathrm{write}$ JSON formatted data.

Between the communication path that enables the interaction of the MG-EMS and the HMI/SCADA there is an SQLdatabase, where the measurements (input data) will be saved waiting to be processed by the MG-EMS and the set-points (output data/commands) will be saved to be processed by the SCADA. To populate the database with new data or to retrieve old data from the database a web REST API is used.

\section{Building EMS at HSB LL}

In the BEMS project, the HSB LL building is seen as an energy hub, where the residents actively participate in demandside management. The aim of the project is to develop a BEMS for HSB LL, with the objective to reduce the total energy cost and the peak demand in the building by controlling the consumption of heating and electricity and the charging/discharging of the batteries (including PEV batteries).

1) BEMS Demonstration: The rule-based and optimizationbased algorithms that are developed and tested in this project aim to activate the energy flexibility of the building. The BEMS uses the flexibility provided by the users by shifting the start time of controllable loads, such as washing machines, dishwashers, tumble dryers and drying cabinets.

In this energy hub, the interaction between electricity and heating loads is also utilized to reduce the stress from the utility distribution networks (district heating and electricity network) by managing the peak demand, while ensuring at the same time that the comfort level of the customers will not be compromised. To create this interaction two air-to-water 9 $\mathrm{kW}$ heat pumps including three 500 litre hot water storage tanks are planned to get installed at the HSB LL. The heating system will consist of the district heating network, the heat pumps and the water storage tanks.

The project is expected to integrate more renewable energy, reduce customers' energy bills, reduce the building's carbon footprints, improve the operation of distribution networks, and

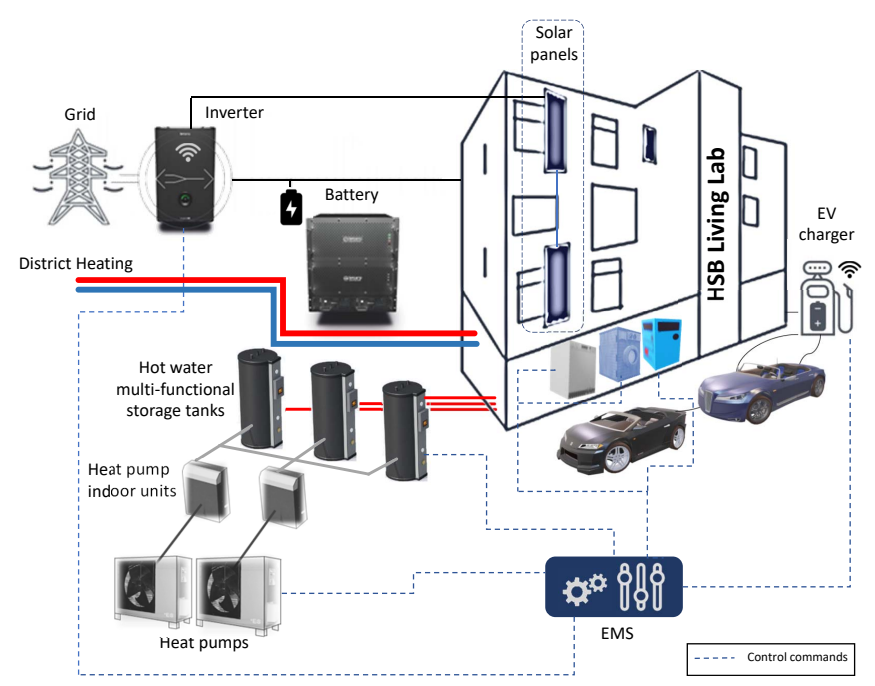

Fig. 4. An overview of the controllable loads at the HSB LL.

reduce the needs for distribution grid investment. The findings provide guidelines and strategies for the utility and building companies to include energy services in their portfolios.

2) Communication Set-up: The future energy demand is forecasted using the historical load data, weather data, and social factors. The forecasted data are used as an input to a centralized BEMS, which operates near to real-time and determines the optimal operation of controllable sources. The control commands are sent to the battery and the laundry machines via MQTT. The components of the heating system will be controlled via Modbus communication. The PEV charging station with two charging points, which is also expected to be installed, will use the open charge point protocol (OCPP) for communication with the BEMS.

Fig. 4 depicts a general overview of the controllable loads at the HSB LL. Currently, the developed BEMS controls the battery storage and the laundry machines. In the future, the BEMS will also control the PEV battery charging and determine the optimal settings of the inverter-controlled heat pumps, the operation of the water storage tanks, and the heat delivered to the building from the district heating network.

\section{UNITED-GRID: Solutions for Intelligent Distribution Grids}

The main aim of UNITED-GRID project is to secure and optimize the operation of future intelligent distribution networks with unprecedented complexity caused by new distributed market actors along with emerging technologies such as renewable generation, energy storage, and demand resources. A task in the project will develop a coordinated voltage control strategy to calculate the optimal set-points for the local controllers of (on-load tap changers) OLTC, BESS and PV.

The developed control strategy will be based on model predictive control (MPC) and supported by real-time measurements. The application of MPC to power systems offers natural handling of multi-variable control problems, ability to observe constraints on inputs and outputs, etc. These advantages, coupled with the fact that MPC is optimization-based, provide 
a great opportunity for its application to increasingly complex control challenges in distribution systems with a large number of PV, BESS and flexible loads providing demand response services. The developed task, which will be demonstrated at the Chalmers testbed, will utilize real-time measurements obtained at different points of the network.

\section{EXAMPle of Remote BATTERy CONTROL}

To integrate the battery dispatch with an emulated EMS preliminary tests were conducted, where the implemented battery control introduced the following improvements:

1) The battery responds to external control signals via the MQTT protocol. This increases the control options compared to the internally implemented control, which is triggered based on a peak and a low power threshold adjustable only by the manufacturer.

2) The proposed control is designed to incorporate forecasted values, which reduces the uncertainties. The default battery operation is based on instantaneous measurements, which may fluctuate rapidly.

3) The amount of dispatched charging power takes into consideration the non-linear dependency on the SOC, something which is often ignored as suggested in [16].

For the remote control, an MQTT client is created for the EMS, which must be connected to the MQTT broker provided by the converter of the PV and BESS system. The converter is coupled with sensors that monitor the DC/DC converters of the solar panels and the battery as well as the building consumption and the power at the point of grid connection.

The MQTT client of the EMS must be subscribed to the relevant topics to read the available measurements. These topics continuously transmit messages with the measurements encoded in JSON format and a data refresh rate of $1 \mathrm{sec}$. The algorithm of the EMS reads, decodes and processes the data and then decides on the power charging/discharging request. The MQTT client publishes the battery request (encoded in JSON) to the topic associated with the battery control. The application of the MQTT protocol, which facilitates this communication, is realised using the MQTT toolbox in MATLAB.

\section{A. Test Purpose and Methodology}

The EMS implements a rule-based algorithm that tries to constrain the power exchange with the grid between a peak and a low load threshold ( $P_{\text {peak }}$ and $P_{\text {low }}$, respectively), which can be externally set by the user/operator. The purpose of this test is to flatten the aggregate power profile as seen by the DSO at the PCC of the PV and BESS system. The algorithm updates the battery output set-point per iteration $i$ based on the average PCC power exchange $\left(P_{i}^{P C C}\right)$ of the previous iteration loop (each loop lasts one minute).

The calculation of the discharging power output request $P_{i}^{-}$ is based on a linear BESS model with a constant discharging power limit $P_{m a x}^{-}$, whereas the charging power output $P_{i}^{+}$is calculated with a use of a piecewise linear BESS, where the charging power limit $P_{\max }^{+}$depends on the battery state-ofcharge $(S O C)$ [16]. To avoid deep discharges/charges of the

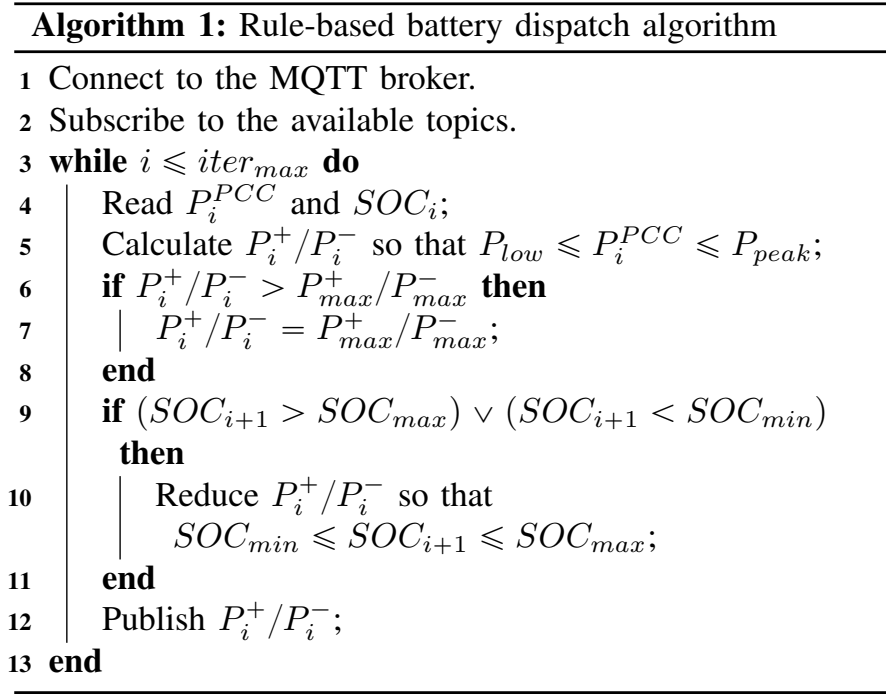

battery, the $S O C$ limits are considered $\left(S O C_{\min }, S O C_{\max }\right)$. Algorithm 1 describes the interaction between the EMS and the controlled system via the MQTT protocol and the proposed methodology.

\section{B. Evaluation of Initial Result}

The response of the battery to the requests (power setpoints) can be seen in Fig. 5. The data exchange with MQTT is very fast and the set-point has been received by the broker in 1 sec. The process of the command by the converter controller of the PV and BESS system takes 10 seconds. After that, the command is transmitted to the battery DC/DC converter through power line communication (PLC), which adds a small delay to the transmission of the set-point. Once the battery management system (BMS) is actuated the set-point is reached in about $10 \mathrm{sec}$ depending on the battery status and the amount of requested power.

The results show a total delay of maximum $30 \mathrm{sec}$, so this system could very well serve the goals of an optimizationbased energy management system with a 5-minute time scale considering forecast, which is the next stage of this demonstration. However, the system's communication/control structure should be upgraded to support the demonstration of grid supporting services such as primary frequency control.

The test results clearly show that the battery power output matches exactly the dispatched set-points. This is very important for the integration of this method with an energy management system because it ensures that the operational objective can be met.

\section{Challenges And Lessons Learned}

The integration to the SCADA and the interoperability of the devices, which is essential to smart grid implementation, has proven to be one of the biggest challenges. This process can be very different for each controllable home device or inverter-embedded controller as they might use different communication protocols (e.g., MQTT, OCPP, Modbus) on top of TCP/IP connection. Moreover, not all functionalities might 


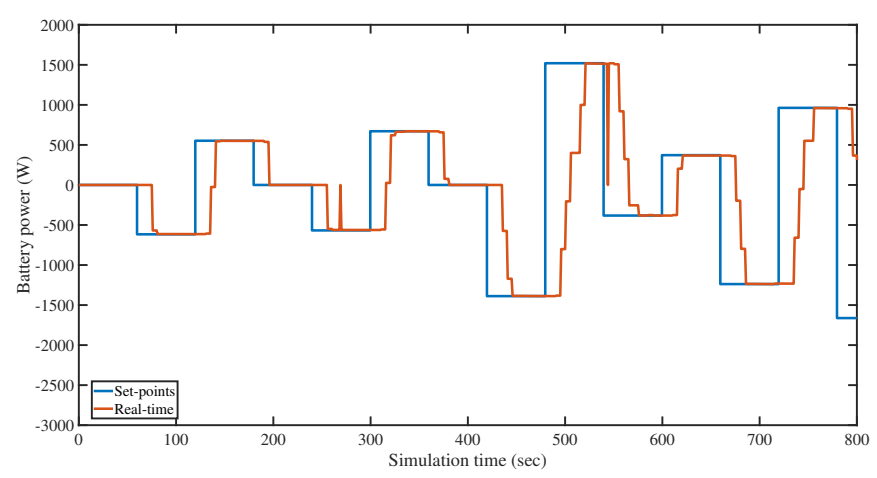

Fig. 5. The set-points and real-time measurements of the battery power.

be available through Internet interface (especially those who allow modification of the control settings). Eventually, additional interfacing devices might have to be used to guarantee interoperability of the control set-up.

The agreement with the stakeholders, that are associated with the physical test site is also a complicated task. The demonstration activities must be carefully planned so that any undesired deviation from expected costs or the level of comfort for the end-users can be ruled out. Moreover, a non-disclosure agreement might also be required for data protection. Above all, the integration of the test systems must comply with the security requirements.

To prevent unauthorized access a secure virtual private network (VPN) connection must be established between the developed models and the on-site control systems. The information security of the control systems can also be improved by blocking all incoming traffic from the Internet.

To achieve this during the MG-EMS demonstration, the MG-EMS stores the control signals in the SQL-database using a web-service. Another service running on the inside of the protected network continuously polls the SQL-database, fetches commands, and forwards them to the SCADA. The SCADA operator can also disable the automated control at any time by shutting down the web-service that fetches commands, which also enhances the technical safety of the control system.

Secure web-based solutions add a small delay in the response, which indicates that they are not compatible with fast coordinated control schemes. This delay is, however, acceptable for the proposed EMS demonstrations, as long as it is considered along with other processing and communication delays to properly define the time of dispatch of the set-points.

\section{CONCLUSIONS}

This paper presents the development of a smart grid testbed at the Chalmers University of Technology campus. The testbed supports the demonstration of many intelligent grid functionalities including energy management strategies at the microgrid and building level, coordinated voltage control, and energy transactions within a local energy market platform.

Emphasis is given on the description of the necessary upgrades of the physical energy system of the university within the scope of the on-going demonstration activities.
Secure web-based solutions, additional flexible resources, and interoperable communication interfaces are part of these adaptations, which are discussed along with identified challenges and gained knowledge.

As an example, a recently developed remote battery control test system is presented. Initial results proved that the proposed control is suitable for EMS applications. Future research directions will provide detailed optimization and simulation models at the smart grid and building level, validated through on-site implementation and field measurements.

\section{ACKNOWLEDGMENT}

The authors acknowledge the contribution of Andreas Jonasson, Mahsa Eshtehardi, and Taz Lodder from Chalmers IT system and services as well as Anders Becker from Ferroamp to the integration of EMS solutions.

\section{REFERENCES}

[1] B. Washom, J. Dilliot, D. Weil, J. Kleissl, N. Balac, W. Torre, and C. Richter, "Ivory tower of power: Microgrid implementation at the university of California, San Diego," IEEE Power Energy Mag., vol. 11, no. 4, pp. 28-32, July/Aug. 2013.

[2] A. Bonfiglio, M. Brignone, F. Delfino, A. Nilberto, and R. Procopio, "Definition and experimental validation of a simplified model for a microgrid thermal network and its integration into energy management systems," Energies, vol. 9, no. 11, p. 914, Nov. 2016.

[3] M. Husein and I.-Y. Chung, "Optimal design and financial feasibility of a university campus microgrid considering renewable energy incentives," Applied energy, vol. 225, pp. 273-289, Sep. 2018.

[4] Y. Zhang and Q.-S. Jia, "A simulation-based policy improvement method for joint-operation of building microgrids with distributed solar power and battery," IEEE Trans. on Smart Grid, vol. 9, no. 6, pp. 6242-6252, Nov. 2018.

[5] Q. Kong, M. Fowler, E. Entchev, H. Ribberink, and R. McCallum, "The role of charging infrastructure in electric vehicle implementation within smart grids," Energies, vol. 11, no. 12, p. 3362, Dec. 2018.

[6] V. M. Larios, L. Gomez, O. B. Mora, R. Maciel, and N. VillanuevaRosales, "Living labs for smart cities: A use case in Guadalajara city to foster innovation and develop citizen-centered solutions," in Proc. IEEE Int. Smart Cities Con. (ISC2), Sep. 2016.

[7] B. Delinchant, F. Wurtz, S. Ploix, J.-L. Schanen, and Y. Marechal, "GreEn-ER living lab: A green building with energy aware occupants," in Proc. 5th Int. Con. on Smart Cities and Green ICT Syst. (SMARTGREENS), Apr. 2016.

[8] D.-H. Shin, "A living lab as socio-technical ecosystem: Evaluating the Korean living lab of internet of things," Government Information Quarterly, vol. 36, no. 2, pp. 264-275, Apr. 2019.

[9] m2M-GRID, "From micro to Mega-GRID: Interactions of micro-grids in active distribution networks." [Online]. Available: https://m2m-grid.eu/

[10] FED, "Fossil free energy districts," [Online] Available: https://www.uiainitiative.eu/en/uia-cities/gothenburg.

[11] UNITED-GRID, "Solutions for intelligent distribution grids," [Online] Available: https://united-grid.eu/.

[12] Chalmers University of Technology, "Innovative energy management system for smart buildings and grid interactions." [Online]. Available: https://www.chalmers.se/en/projects/Pages/Innovativeenergy-management-system-for-smart-buildings-andgrid.aspx

[13] K. Antoniadou-Plytaria, T. Tam Mai, G. Mouloud, "Preparation for demonstration sites - report for m2M-GRID project," 2019. [Online]. Available: https://m2m-grid.eu/results/

[14] HSB, "HSB living lab." [Online]. Available: https://www.hsb.se/hsblivinglab/

[15] A. Capozzoli, M. S. Piscitelli, S. Brandi, D. Grassi, and G. Chicco, "Automated load pattern learning and anomaly detection for enhancing energy management in smart buildings," Energy, vol. 157, pp. 336-352, Aug. 2018.

[16] H. Pandžić and V. Bobanac, "An accurate charging model of battery energy storage," IEEE Trans. on Power Syst., vol. 34, no. 2, pp. 14161426, Mar. 2019. 